\title{
Effect of autoimmune diseases on risk and survival in histology-specific lung cancer
}

\author{
Kari Hemminki**\#, Xiangdong Liü, Jianguang $\mathrm{Ji}^{\#}$, \\ Jan Sundquist*, and Kristina Sundquist ${ }^{*}$
}

ABSTRACT: Patients with autoimmune diseases are at ancreased risk of cancer due to underlying dysregulation of the immune system or treatment. Data on cancer incidence, mortality and survival after autoimmune diseases would provide further information on the clinical implications.

We systematically analysed data on lung cancer in patients diagnosed with 33 different autoimmune diseases. Standardised incidence ratios (SIRs), standardised mortality ratios (SMRs) and hazard ratios (HRs) were calculated for subsequent incident lung cancers or lung cancer deaths up to 2008 in patients hospitalised for autoimmune disease after 1964.

Increased risks of lung cancer were recorded for SIRs after 12 autoimmune diseases, SMRs after 11 autoimmune diseases and HRs after two autoimmune diseases. The highest SIRs and SMRs, respectively, were seen after discoid lupus erythematosus (4.71 and 4.80), polymyosistis/ dermatomyositis (4.20 and 4.17), systemic lupus erythematosus (2.47 and 2.69), rheumatic fever (2.07 and 2.07) and systemic sclerosis (2.19 and 1.98). Autoimmune disease did not influence survival overall but some autoimmune diseases appeared to impair survival in small cell carcinoma.

All autoimmune diseases that had an SIR $>\mathbf{2 . 0}$ are known to present with lung manifestations, suggesting that the autoimmune process contributes to lung cancer susceptibility. The data on survival are reassuring that autoimmune diseases do not influence prognosis in lung cancer.

KEYWORDS: Comorbidity, immune disturbance, prognosis, risk, treatment

$\mathbf{A}$ utoimmune diseases constitute many disease manifestations which jointly affect $5-10 \%$ of the population in developed countries [1]. Autoimmune diseases are characterised by the activation of T-cells or B-cells, or both, in the absence of an ongoing infection. The normal function of these cells is to recognise specific foreign antigens, but they also possess a low level of autoreactivity towards self- or autoantigens. In autoimmune diseases, autoreactivity is increased either systemically or organ-specifically [2]. Many cancers, for reasons that are not known, are increased in autoimmune disease patients [3-6]. The immunological disturbances in autoimmune diseases may be an important mechanism in cancer formation, but immunosuppressive therapy may also contribute [7].

Lung and pleural manifestations of systemic autoimmune disease are heterogeneous [8]. They may not only affect lung parenchyma, but may involve the conducting airways, respiratory muscles and pulmonary vasculature. Common features of disease presentation are pleuritis with effusion and interstitial progressive lung disease. Pleural effusions are also caused by infections, which need to be excluded. Rheumatoid nodules in the airways may mimic neoplastic growth and require differential diagnostics. Pleuropulmonary disease is a common feature of systemic lupus erythematosis, rheumatoid arthritis, systemic sclerosis, polymyositis/dermatomyositis, Sjögren's syndrome, granulomatosis with polyangiitis (Wegener's) and sarcoidosis [8].

Lung cancer risks have been associated with systemic lupus erythematosis, rheumatoid arthritis, Crohn's disease, psoriasis and sarcoidosis [914]. No increase has been observed in ulcerative colitis patients [15]. Some studies have analysed survival in lung cancer after an autoimmune disease; decreased survival was noted after sarcoidosis, Crohn's disease and ulcerative colitis, but no effect was reported after psoriasis and rheumatoid arthritis [16-19].

In the present study we examined the risk of lung cancer (standardised incidence ratio (SIR)), mortality in lung cancer (standardised mortality ratio (SMR)) and survival in lung cancer (hazard ratio

\section{AFFILIATIONS}

${ }^{*}$ Division of Molecular Genetic Epidemiology, German Cancer Research Center (DKFZ), Heidelberg, Germany.

${ }^{\#}$ Center for Primary Health Care Research, Lund University, Malmö, Sweden.

'Stanford Prevention Research Center, Stanford University School of Medicine, Stanford, CA, USA.

CORRESPONDENCE

K. Hemminki

Division of Molecular Genetic

Epidemiology

German Cancer Research Center (DKFZ)

Im Neuenheimer Feld 580

D-69120 Heidelberg

Germany

E-mail: k.hemminki@dkfz.de

Received:

Dec 192011

Accepted after revision:

Jan 232012

First published online:

Feb 092012 
(HR)) in patients who had been hospitalised for any one of 33 autoimmune diseases, in the total Swedish population. Lung cancer manifests in various histological types and it is of interest to assess the various outcome measures by histology, for which hardly any data are available. Many autoimmune diseases show large sex differences and, therefore, a sexspecific analysis was also included.

\section{MATERIAL AND METHODS}

The research database used in the present study is a subset of the national MigMed 2 datasets from the Center for Primary Health Care Research, Lund University, Malmö, Sweden. It has been used in our previous studies on autoimmune disease and various cancers, whereby the methods used have been identical, except for the cancer under study [6, 20, 21]. Autoimmune disease patients were identified from the Swedish Hospital Discharge Register which records complete data on all discharges with dates of hospitalisation and diagnoses in some regions since 1964 and nationwide since 1986. The International Classification of Diseases codes have been described previously $[22,23]$. A total of 33 diseases were covered. However, seven of them had less than 11 cases for lung cancer, therefore, data are not shown for autoimmune haemolytic anaemia, chorea minor, lupoid hepatitis, polyarteritis nodosa, polymyalgia rheumatic, Reiter's disease and type 1 diabetes; these diseases were not significantly associated with lung cancer. The linkages were performed using an individual national identification number that is assigned to each person in Sweden for life. This number was replaced by a serial number for each person in order to provide anonymity.

Cancer data were obtained from the nationwide Swedish Cancer Registry. Person-yrs of follow-up were calculated from date of discharge with the first main diagnosis of autoimmune disease until death, emigration or closing date, December 31, 2008. SIRs were calculated as the ratio of observed to expected number of lung cancers. SMRs were similarly calculated as the ratio of observed to expected number of cancer-specific deaths. Expected numbers were calculated for anyone not hospitalised for any autoimmune disease. The expected numbers were calculated as age (5-yr groups), sex, period (5-yr groups), region and socioeconomic status-specific standard incidence rates. Additional adjustments were made for hospitalisation for obesity using codes: ICD-7 287.00, 287.09; ICD-8 277.99; ICD-9 278A; and ICD-10 E65-E68. A total of 30,020 individuals had been hospitalised for obesity. Similar adjustments were made for hospitalisation for chronic obstructive pulmonary disease (COPD) (as a surrogate for smoking), with 260,243 individuals affected (codes: ICD-7 500502; ICD-8 490-493; ICD-9 490-496; and ICD-10 J40-J49) and alcoholism (codes: ICD-9 303; and ICD-10 F10.1-F10.9), with 181,862 individuals affected. Confidence intervals were calculated assuming a Poisson distribution. The Cox regression analyses were used to estimate HRs, which could be interpreted as mortality rate ratio. Adjustments were performed as above. The analyses were for deaths in lung cancer while deaths from other causes were censored. The reference population was anyone not hospitalised for any autoimmune disease, as for SIR and SMR. The proportional hazard assumption for the covariates was tested by Schoenfeld residuals and by plotting the log of the negative log of the survival function versus the log of time. The study was approved by the regional ethical review board at Lund University.

\section{RESULTS}

The total number of autoimmune disease patients was 402,062 and they accumulated 4.8 million person-yrs at risk (mean follow-up 11.9 yrs). Table 1 shows the numbers of patients for each autoimmune disease; the largest individual disease was rheumatoid arthritis with 72,309 patients. Of the 33 autoimmune diseases analysed, 26 had more than 10 cases of lung cancers and these are shown in table 1. For lung cancer after any autoimmune disease, including the ones not shown, SIR was 1.33, SMR was 1.26 and HR was 1.02 (not significant). SIRs (12 increases, one decrease) and SMRs (11 increases, one decrease) correlated and, even though neither was statistically significant, the direction (increase or decrease) was almost always the same (table 1). For this reason SMR are only shown in table 1 . The highest SIRs and SMRs, respectively, were seen after discoid lupus erythematosus (4.71 and 4.80), polymyosistis/dermatomyositis (4.20 and 4.17), systemic lupus erythematosus (2.47 and 2.69), rheumatic fever (2.07 and 2.07) and systemic sclerosis (2.19 and 1.98). SIR was decreased in pernicious anaemia patients (0.70). Only after amyotrophic lateral sclerosis was HR increased (1.60); patients with coeliac disease (0.49) and rheumatic fever (0.76) showed favourable survival.

None of the individual SIRs differed significantly (95\% confidence interval overlapping) by sex (table 2), nor did the overall SIRs (1.29 for females and 1.36 for males). SIRs for lung cancer after Crohn's disease were only significant for females and those after chronic rheumatic heart disease, Hashimoto's disease/hypothyroidism and sarcoidosis were only significant for males. The only significant $\mathrm{HR}$ for females was for pernicious anaemia patients (2.12), for males it was 1.03, but the difference was not significant. Males with polymyositis/ dermatomyositis (HR 1.72) and rheumatoid arthritis (HR 1.15) had decreased survival. Male coeliac disease patients showed a favourable survival (HR 0.50).

Table 3 presents results according to the four main histological types. The overall case numbers were approximately equal for adenocarcinoma $(n=741)$ and for squamous cell carcinoma $(n=740)$, which were double that of small cell carcinoma $(n=388)$; large cell carcinoma had intermediate case numbers $(n=615)$. The overall SIRs increased after autoimmune disease for all histological types, but mostly for small cell (1.43) and squamous cell (1.40) carcinoma. Squamous cell carcinoma was increased after nine autoimmune diseases, large cell carcinoma after eight autoimmune diseases, small cell carcinoma after seven autoimmune diseases and adenocarcinoma after five autoimmune diseases. All types of lung cancer were increased after discoid lupus erythematosus and the highest risk was for small cell carcinoma (7.36, the highest risk in the study). Similarly, all lung cancer histologies were increased after polymyosistis/dermatomyositis, systemic lupus erythematosus, rheumatoid arthritis and psoriasis; however, for the latter two, the adenocarcinoma increase was of borderline significance. Again, none of the SIRs differed significantly (95\% confidence intervals overlapping) between the histological types. Squamous cell carcinoma was only increased after primary biliary cirrhosis (5.10). Adenocarcinoma and squamous cell carcinoma were increased after rheumatic fever and systemic sclerosis. Large cell carcinoma and small cell carcinoma were increased after thyroid disease. The only significant decrease in SIR was 0.40 for adenocarcinoma after pernicious anaemia. 


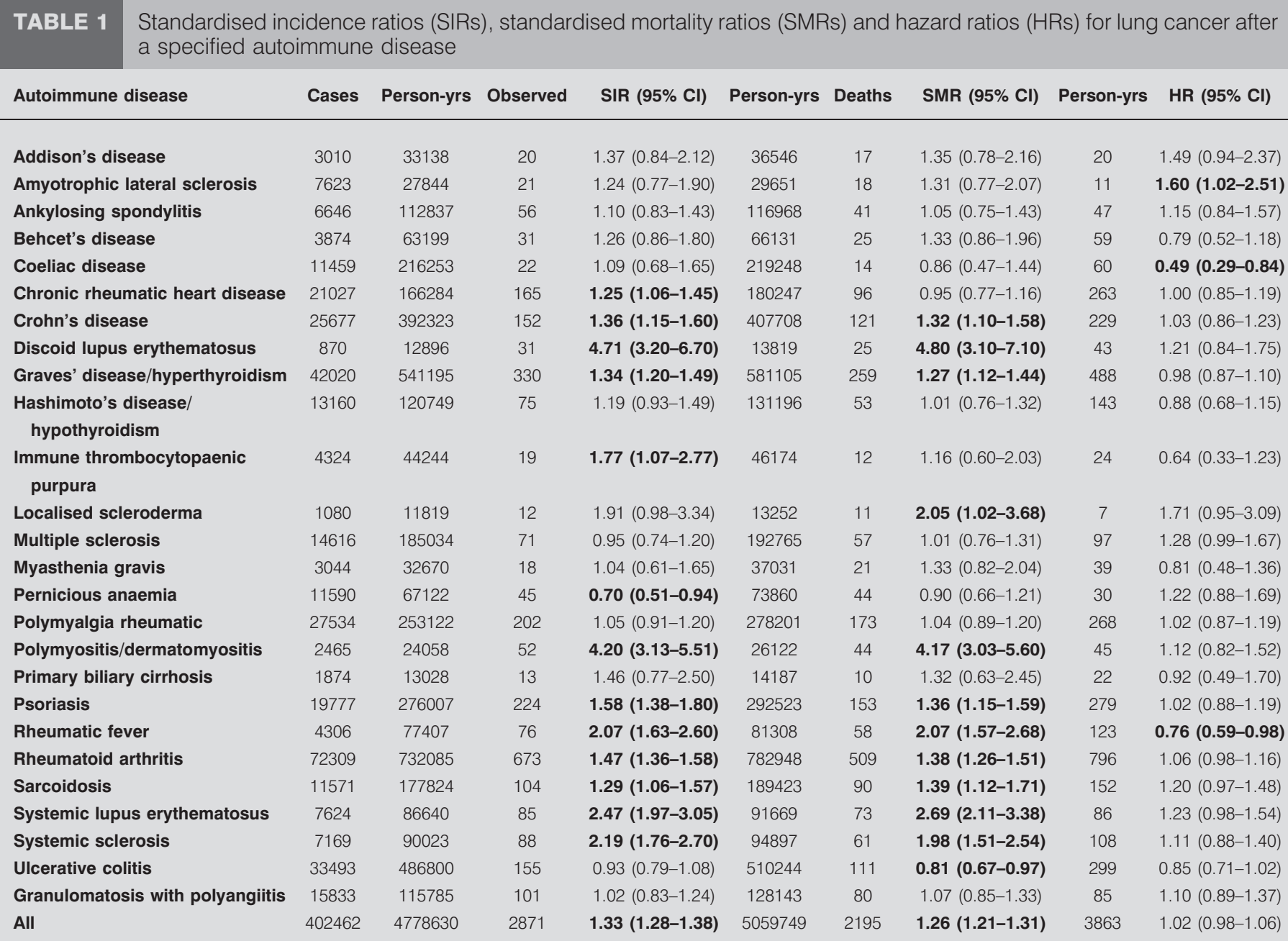

Data are presented as $\mathrm{n}$, unless otherwise specified. Bold indicates that the $95 \% \mathrm{Cl}$ does not include 1.00 .

As shown in table 3, the overall HRs were close to unity for all histologies except for large cell carcinoma (1.13). Survival did not differ from the reference group for any autoimmune disease patients diagnosed with adenocarcinoma or squamous cell carcinoma. Rheumatic polymyalgia and psoriasis had a decreased survival in large cell carcinoma (HR 1.51 and 1.39, respectively). Small cell carcinoma showed impaired survival after five autoimmune diseases, worst after amyotrophic lateral sclerosis (HR 5.49) and discoid lupus erythematosus (HR 2.83).

\section{DISCUSSION}

The present study of 402,062 autoimmune disease patients showed an increased risk of lung cancer for patients with 12 different autoimmune diseases. The overall SIR was 1.33 and the risk was increased after the common autoimmune diseases, rheumatoid arthritis (1.47), Graves' disease/hyperthyroidism (1.34) and Crohn's disease (1.36). This is the largest published study and the only one comparing systematically risk (SIR), mortality (SMR) and survival (HR). As lung cancer is a fatal disease, SMRs correlated closely with SIRs. Autoimmune disease did not impair overall survival, for which the HR was 1.02. Diagnoses of autoimmune diseases, based on discharge data, are of high quality as hospitalised patients are seen by many doctors, often in specialist departments. A recent review on the validity aspects of the Swedish Hospital Discharge Register reported diagnostic accuracy (positive predictive values) for various autoimmune diseases, ranging from $74 \%$ for inflammatory bowel disease to $96 \%$ for rheumatoid arthritis [24].

Coverage is another issue that depends, for example, on the severity of the condition. The aforementioned review cited $42.5 \%$ for rheumatoid arthritis as the only autoimmune disease [24]. We have discussed coverage in many of our previous papers on familial clustering of autoimmune diseases [25-28]. Severe debilitating conditions, such as Graves' disease, type 1 diabetes mellitus, Crohn's disease and ulcerative colitis lead to hospitalisation at one point or another in Sweden. In contrast, Hashimoto's disease/hypothyroidism is an example of a disease with a low degree of hospitalisation. The Swedish Cancer Registry records all new cases of cancer and close to $100 \%$ of the cases are histologically or cytologically confirmed.

An unavoidable issue in the study of lung cancer is smoking, and whether it is a confounder in the present study. If smoking caused an autoimmune disease then the present observations 


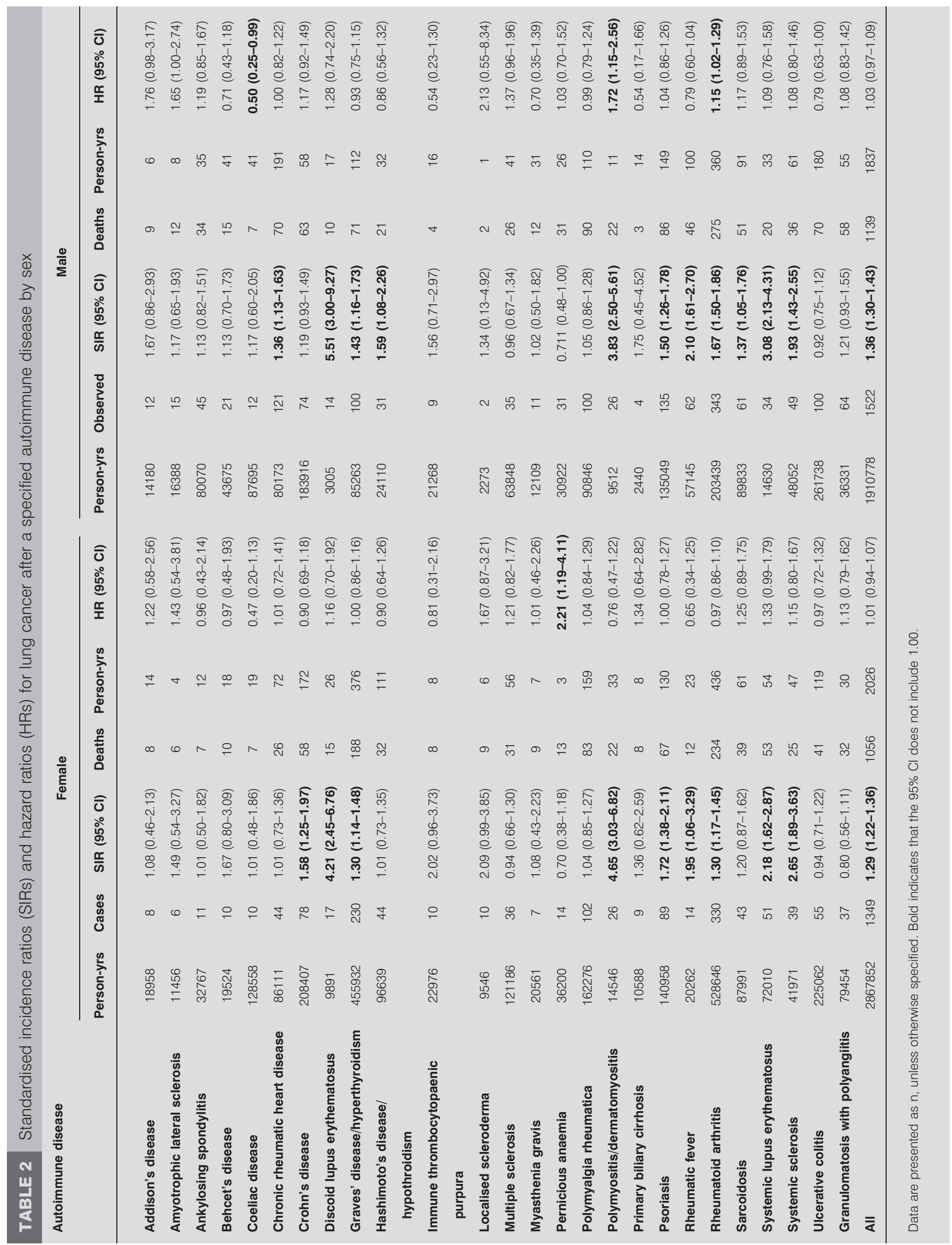




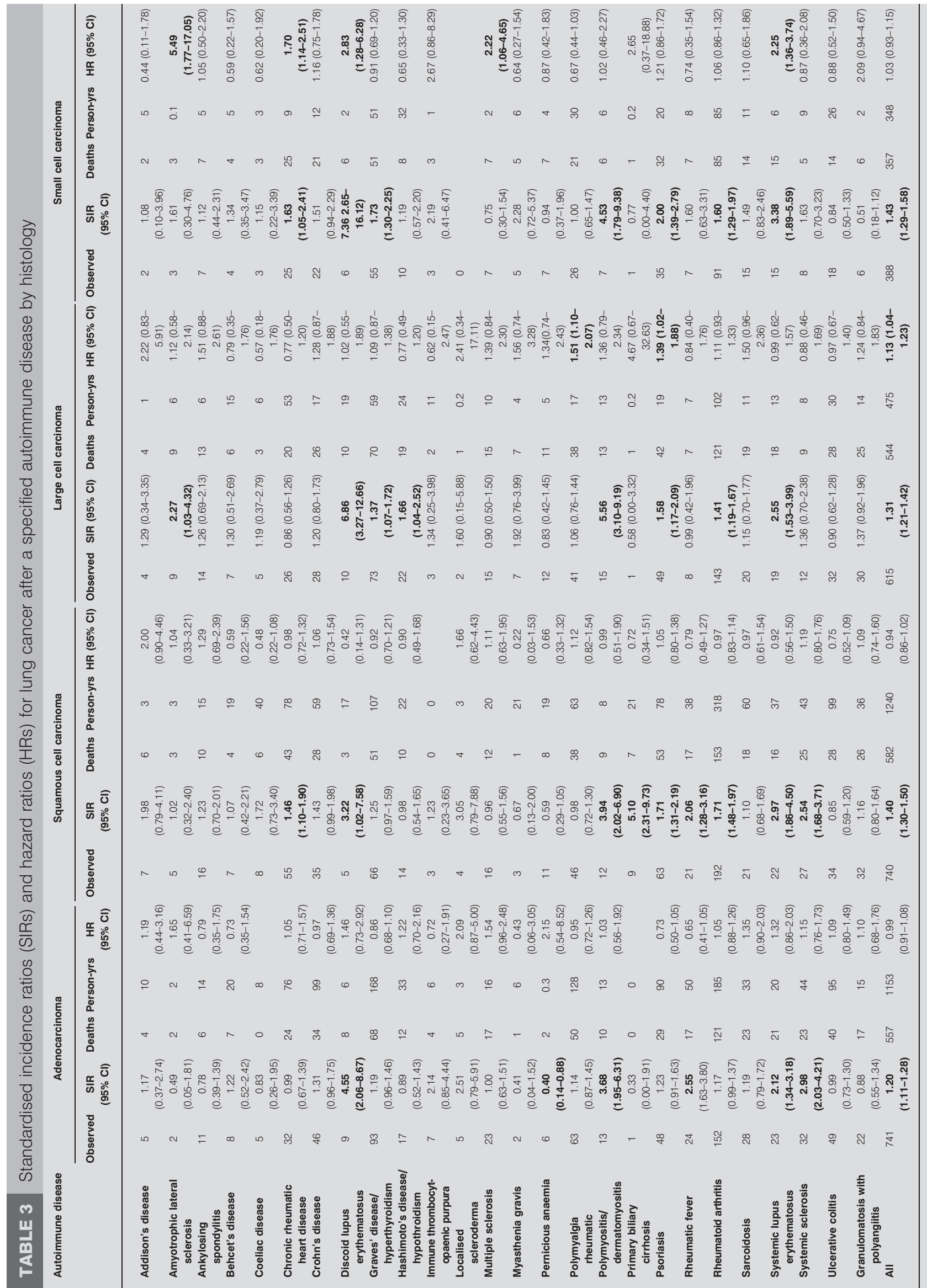


could be ascribed merely to smoking. Smoking is a known risk factor for some autoimmune diseases, including rheumatoid arthritis, systemic lupus erythematosus, Graves' disease and primary biliary cirrhosis, but for many other autoimmune diseases data on smoking are either lacking or controversial [29]. We adjusted the data for COPD which is, of course, not optimal, but the only available means, together with socioeconomic status, to control for the possible bias due to differential smoking habits. However, to explain, even without any adjustments, the observed differences by smoking habit would require an extreme segregation of smoking behaviour [30]. In addition, the practically identical SIRs between males and females rule out strong effects of smoking because historically smoking prevalence has been higher among Swedish males compared to females and the female lung cancer incidence has not yet reached the male incidence [31]. A further point is that survival may be worse among smokers than nonsmokers, and the present overall HR was $1.02[32,33]$.

Interestingly, all autoimmune diseases that were associated with an SIR $>2.0$ are known to present with various kinds of lung manifestations. The highest risk was observed after discoid lupus erythematosus (4.71); generalised discoid lupus can affect the lungs and it can progress to systemic lupus erythematosus with common lung manifestations. The present SIR for systemic lupus erythematosus was 2.47. An excess in lung cancer has been observed in six small previous studies but no specific histology has been implicated $[9,10]$. Herein we show that both discoid lupus erythematosus and systemic lupus erythematosus affect all lung cancer histologies, with the highest risk for small cell carcinoma. The present risk for lung cancer in systemic sclerosis patients was 2.19, somewhat higher than the 1.6 obtained in a Danish study applying a similar design as our study [34]. The localised form of the disease, localised scleroderma, showed a somewhat lower SIR but higher SMR. Both of these autoimmune diseases were approximately four times more common in females compared to males and the SIRs were also somewhat higher among females. Among other autoimmune diseases with lung manifestations, rheumatoid arthritis and sarcoidosis showed an increased risk of lung cancer, in agreement with previous studies [11, 14], whereas no increased risk was noted for Sjögren's syndrome or granulomatosis with polyangiitis [8]. The high risks of lung cancer in diseases that show local lung manifestations suggest that autoimmune lesions may help to drive lung cancer. Similarly, high risks of localised cancers have been described for Crohn's disease and ulcerative colitis [35,36]. These data support the role of localised inflammation and related oxidative lesions in promoting cancer $[37,38]$. Accordingly, lung cancer mortality is significantly reduced in clinical trials with daily intake of aspirin [39]. Although we cannot exclude the role of immunosuppressive therapy on lung cancer risk after some autoimmune diseases, there was no increase, for example, after myasthenia gravis for which immunosuppressive therapy may be applied for extended periods of time [40,41].

The interpretation of the histology-specific analyses is complicated because of small numbers and changing trends in histology. It was, however, striking that the rarest histological type, small cell carcinoma, showed the highest SIR for discoid lupus erythematosus (7.36) and for the related systematic lupus (3.38). Small cell carcinoma showed also the highest overall risks. Also of note was that among the 13 cases of lung cancers following primary biliary cirrhosis, nine were squamous cell carcinomas (SIR 5.10).

The data on survival showed that autoimmune disease has no large influence, implying that autoimmune disease does not limit treatment even for autoimmune diseases that have pulmonary manifestations, and that the autoimmune process does not facilitate lung cancer progression. One of the reasons may be the poor prognosis of lung cancer in itself. In our previous study, survival from seven digestive tract cancers was followed after autoimmune disease [20]. HRs were increased for many cancers with an intermediary prognosis but not for stomach cancer, which has a poor prognosis in itself. It is noteworthy in the present study that there were only four autoimmune disease cancer pairs for which both SIR and HR were increased and three of these included small cell carcinoma after chronic rheumatic heart disease, discoid lupus erythematosus and the related systematic lupus. As the principle treatment for small cell carcinoma is chemotherapy, compared to surgery for other types, it is conceivable that comorbidities are more treatment limiting when the treatment lasts for an extended period.

In conclusion, the present data showed an increase in SIR for lung cancer after 12 autoimmune diseases, SMR was increased after 11 autoimmune diseases and HR after two autoimmune diseases. All autoimmune diseases associated with an SIR $>2.0$ are known to present with lung manifestations, suggesting that the autoimmune process contributes to lung cancer susceptibility. Autoimmune disease did not influence survival overall but some autoimmune diseases appeared to impair survival in small cell carcinoma.

\section{SUPPORT STATEMENT}

This study was supported by the Swedish Council for Working Life and Social Research (Stockholm, Sweden) and Deutsche Krebshilfe (Cologne, Germany).

\section{STATEMENT OF INTEREST}

None declared.

\section{REFERENCES}

1 Forabosco P, Bouzigon E, Ng MY, et al. Meta-analysis of genomewide linkage studies across autoimmune diseases. Eur J Hum Genet 2009; 17: 236-243.

2 Zhernakova A, van Diemen CC, Wijmenga C. Detecting shared pathogenesis from the shared genetics of immune-related diseases. Nat Rev Genet 2009; 10: 43-55.

3 Goldin LR, Landgren O. Autoimmunity and lymphomagenesis. Int J Cancer 2009; 124: 1497-1502.

4 Brown LM, Gridley G, Check D, et al. Risk of multiple myeloma and monoclonal gammopathy of undetermined significance among white and black male United States veterans with prior autoimmune, infectious, inflammatory, and allergic disorders. Blood 2008; 111: 3388-3394.

5 Landgren AM, Landgren O, Gridley G, et al. Autoimmune disease and subsequent risk of developing alimentary tract cancers among 4.5 million US male veterans. Cancer 2011; 117: 1163-1171.

6 Hemminki K, Liu X, Ji J, et al. Autoimmune disease and subsequent digestive tract cancer by histology. Ann Oncol 2012; 23: 927-933.

7 Vajdic CM, van Leeuwen MT. Cancer incidence and risk factors after solid organ transplantation. Int J Cancer 2009; 125: 1747-1754. 
8 Rayner CF, Grubnic S. Pulmonary manifestations of systemic autoimmune disease. Best Pract Res Clin Rheumatol 2004; 18: 381-410.

9 Bernatsky S, Ramsey-Goldman R, Clarke A. Malignancy and autoimmunity. Curr Opin Rheumatol 2006; 18: 129-134.

10 Gayed M, Bernatsky S, Ramsey-Goldman R, et al. Lupus and cancer. Lupus 2009; 18: 479-485.

11 Hemminki $\mathrm{K}, \mathrm{Li}$ X, Sundquist $\mathrm{K}$, et al. Cancer risk in hospitalized rheumatoid arthritis patients. Rheumatology (Oxford) 2008; 47: 698-701.

12 Hemminki K, Li X, Sundquist J, et al. Cancer risks in Crohn disease patients. Ann Oncol 2009; 20: 574-580.

13 Ji J, Shu X, Sundquist K, et al. Cancer risk in hospitalised psoriasis patients: a follow-up study in Sweden. Br J Cancer 2009; 100: 1499-1502.

14 Ji J, Shu X, Li X, et al. Cancer risk in hospitalized sarcoidosis patients: a follow-up study in Sweden. Ann Oncol 2009; 20: 1121-1126.

15 Hemminki K, Li X, Sundquist J, et al. Cancer risks in ulcerative colitis patients. Int J Cancer 2008; 123: 1417-1421.

16 Shu X, Ji J, Sundquist K, et al. Survival in cancer patients with previous hospitalization for sarcoidosis: a Swedish populationbased cohort study during 1964-2006. Ann Oncol 2011; 22: $1427-1434$.

17 Shu X, Ji J, Sundquist J, et al. Survival in cancer patients hospitalized for inflammatory bowel disease in Sweden. Inflamm Bowel Dis 2011; 17: 816-822.

18 Ji J, Liu X, Sundquist K, et al. Survival of cancer in patients with rheumatoid arthritis: a follow-up study in Sweden of patients hospitalized with rheumatoid arthritis 1 year before diagnosis of cancer. Rheumatology (Oxford) 2011; 50: 1513-1518.

19 Shu X, Ji J, Sundquist J, et al. Survival in cancer patients hospitalized for psoriasis: a population-based cohort study in Sweden. Br J Dermatol 2011; 165: 129-136.

20 Hemminki K, Liu X, Ji J, et al. Effect of autoimmune diseases on mortality and survival in subsequent digestive tract cancers. Ann Oncol 2012; 23: 2179-2184.

21 Hemminki K, Liu X, Ji J, et al. Effect of autoimmune diseases on risk and survival in female cancers. Gynecol Oncol 2012; 127: 180-185.

22 Hemminki K, Li X, Sundquist K, et al. Familial risks for chronic obstructive pulmonary disease among siblings based on hospitalizations in Sweden. I Epidemiol Community Health 2008; 62: 398-401.

23 Hemminki K, Li X, Sundquist K, et al. Familial risks for asthma among twins and other siblings based on hospitalizations in Sweden. Clin Exp Allergy 2007; 37: 1320-1325.

24 Ludvigsson JF, Andersson E, Ekbom A, et al. External review and validation of the Swedish national inpatient register. BMC Public Health 2011; 11: 450.
25 Hemminki K, Li X, Sundquist J, et al. Familial associations of rheumatoid arthritis with autoimmune disorders and related conditions. Arthritis Rheum 2009; 60: 661-668.

26 Hemminki K, Li X, Sundquist J, et al. Familial association between type 1 diabetes and other autoimmune and related diseases. Diabetologia 2009; 52: 1820-1828.

27 Hemminki K, Li X, Sundquist J, et al. The epidemiology of Graves' disease: evidence of a genetic and an environmental contribution. J Autoimmun 2010; 34: J307-J313.

28 Hemminki K, Li X, Sundquist K, et al. Familial association of inflammatory bowel diseases with other autoimmune and related diseases. Am J Gastroenterol 2010; 105: 139-147.

29 Costenbader KH, Karlson EW. Cigarette smoking and autoimmune disease: what can we learn from epidemiology? Lupus 2006; 15: 737-745.

30 Lorenzo Bermejo J, Hemminki K. Familial lung cancer and aggregation of smoking habits: a simulation of the effect of shared environmental factors on the familial risk of cancer. Cancer Epidemiol Biomarkers Prev 2005; 14: 1738-1740.

31 Center for Epidemiology. Cancer Incidence in Sweden 2009. Stockholm, The National Board of Health and Welfare, 2010.

32 Belani CP, Marts S, Schiller J, et al. Women and lung cancer: epidemiology, tumor biology, and emerging trends in clinical research. Lung Cancer 2007; 55: 15-23.

33 Parsons A, Daley A, Begh R, et al. Influence of smoking cessation after diagnosis of early stage lung cancer on prognosis: systematic review of observational studies with meta-analysis. BMJ 2010; 340: b5569.

34 Olesen AB, Svaerke C, Farkas DK, et al. Systemic sclerosis and the risk of cancer: a nationwide population-based cohort study. $\mathrm{Br} \mathrm{J}$ Dermatol 2010; 163: 800-806.

35 Sartor R. Mechanisms of disease: pathogenesis of Crohn's disease and ulcerative colitis. Nature Clin Pract Gastroenterol Hepatol 2006; 3: 390-407.

36 Schreiber S, Rosenstiel P, Albrecht M, et al. Genetics of Crohn disease, an archetypal inflammatory barrier disease. Nat Rev Genet 2005; 6: 376-388.

37 Schroder K, Tschopp J. The inflammasomes. Cell 2010; 140: 821-832.

38 Terzic J, Grivennikov S, Karin E, et al. Inflammation and colon cancer. Gastroenterology 2010; 138: 2101-2114 e5.

39 Rothwell PM, Fowkes FG, Belch JF, et al. Effect of daily aspirin on long-term risk of death due to cancer: analysis of individual patient data from randomised trials. Lancet 2011; 377: 31-41.

40 Citterio A, Beghi E, Millul A, et al. Risk factors for tumor occurrence in patients with myasthenia gravis. J Neurol 2009; 256: 1221-1227.

41 Tsinzerling N, Lefvert AK, Matell G, et al. Myasthenia gravis: a long term follow-up study of Swedish patients with specific reference to thymic histology. J Neurol Neurosurg Psychiatry 2007; 78: 1109-1112. 